[pp.41-71]

Vol. 4(1), Enero - Junio, 2016

http://dx.doi.org/10.15359/rnh.4-1.3

\title{
Las relaciones intergeneracionales en la construcción de una sociedad para todas las edades
}

\section{Intergenerational Relationships to Construct a Society for All Ages}

Idalia Alpízar Jiménez

Universidad Nacional

Costa Rica

idalia.alpízar.jiménez@una.cr

\section{Resumen}

El objetivo de este trabajo es difundir una de las primeras experiencias de relaciones intergeneracionales iniciadas en Costa Rica a finales del año 1999; y, al mismo tiempo, proponer que este tipo de experiencias se sigan desarrollando con miras a garantizar un envejecimiento activo dentro de un marco de derechos humanos. El proyecto aquí descrito, con el cual se dieron los primeros pasos en la realización de actividades intergeneracionales en nuestro país, estuvo orientado a propiciar el fortalecimiento de las relaciones entre personas adultas mayores, niños y niñas, jóvenes y personas adultas, para contribuir a disminuir la discriminación hacia estas personas $\mathrm{y}$, con ello, generar las condiciones de una sociedad para todas las edades. A mayor longevidad, mayor tiempo tienen las diversas generaciones para compartir. De ahí que el objetivo de este proyecto haya sido el de fortalecer los vínculos entre diferentes generaciones y reducir brechas, a través de la creación de espacios de encuentro e intercambio, y, mediante un proceso educativo, propiciar la inclusión social de las personas adultas mayores de una manera más efectiva. Así será posible educar para un envejecimiento más saludable, a través de los puentes comunicacionales entre genera-

ciones, y erradicar prejuicios y estereotipos en torno a la vejez y el envejecimiento. 
Palabras clave: Encuentros intergeneracionales, vejez, envejecimiento, derechos humanos, personas adultas mayores, sociedad para todas las edades, mitos y estereotipos.

\begin{abstract}
This paper aims to publicize one of the first intergenerational relationship experiences started in Costa Rica by the end of 1999. Likewise, it proposes the development of this kind of experience in order to guarantee an active aging within the framework of Human Rights. The project here described (which carried out the first intergenerational activities in Costa Rica) aimed to promote strengthening of relationships between older people, children and adults, in order to reduce discrimination against these people, and to create conditions for a society for all ages. The greater the longevity, the longer the time different generations have to share. In consequence, the purpose of this project has been to strengthen the relationship between different generations, and to reduce the gap through the creation of spaces for meeting and exchange, and with an education process to promote more effective social inclusion of older people. Then, educating for healthier aging would be possible through communication bridges between generations and the eradication of prejudice and stereotypes about old age and aging.
\end{abstract}

Keywords: Intergenerational meetings, old age, aging, human rights, older people, society for all ages, myths and stereotypes.

\title{
Antecedentes
}

El inicio del interés por defender los derechos humanos de las personas adultas mayores data del año 1969, en el seno de las Naciones Unidas, momento en el cual se empezaron a emitir una serie de resoluciones que se concretaron en la Asamblea Mundial de Naciones Unidas sobre Envejecimiento, celebrada en 1982.

Veinte años después se realizó la segunda Asamblea Mundial en Madrid, en abril del 2002, en la cual se gestaron la Declaración Política y el Plan de Acción Internacional sobre el Envejecimiento.

Este plan constituía un norte para enfrentar el reto del envejecimiento en el siglo XXI, el cual pasaría a ser una cuestión de primordial importancia, sobre todo en los países en desarrollo, que según se proyectaba, envejecerían rápidamente en la primera mitad del siglo XXI, al punto de que para el 2050 el porcentaje de personas de edad aumentaría considerablemente. 
La revolución de la longevidad, señalada por el Dr. Alexandre Kalache, implicaba que para las últimas seis décadas a nivel mundial, la población de más de 60 años aumentaría de un $8 \%$ a un $10 \%$, y en las próximas cuatro décadas de 10 a 22\%. (Consejo Nacional de la Persona Adulta Mayor, 2013, p. 14)

Por tanto, el plan se centraba en tres áreas fundamentales: las personas de edad y el desarrollo, el fomento de la salud y el bienestar en la vejez, y la creación de un entorno propicio y favorable. Se constituía en el punto de partida para que la formulación de políticas para gobiernos y organizaciones no gubernamentales reorientaran la manera en que sus sociedades percibieran a los ciudadanos y ciudadanas de edad, se relacionaran con estos grupos y les dieran las atenciones.

- Los acelerados cambios demográficos proyectados hacían perentorio que el desarrollo social y económico estuviera orientado hacia una sociedad más integrada desde el punto de vista de la edad, en la que se erradicara la discriminación y la segregación por motivos de edad y se alentara la solidaridad y el apoyo mutuo entre las generaciones.

- Este cambio demográfico planteaba importantes retos en materia de recursos, ya que, por ejemplo, en el caso de los países desarrollados que habían logrado envejecer de forma gradual, se habían tenido que enfrentar problemas resultantes de la relación entre el envejecimiento y el desempleo y la sostenibilidad de los sistemas de pensiones. En el caso de los países en desarrollo las cosas serían más complicadas, por cuanto tenían que afrontar el problema de un desarrollo simultáneo con el envejecimiento de la población.

En ese contexto, las Naciones Unidas proponen en 1999 la idea de construir una sociedad para todas las edades, donde las políticas y planes se ajusten a las necesidades y capacidades de todas las personas, en beneficio de toda la población. Aunque este concepto ya había sido presentado en 1995 en la Cumbre Mundial sobre Desarrollo Social en la que se destacaba, entre otras cosas, el respeto a los derechos humanos, libertades y necesidades especiales de grupos vulnerables y desfavorecidos.

En la referencia que hacen las Naciones Unidas al término, en 1999 se destaca que una sociedad para todas las edades es aquella donde se ajustan sus estructuras, funcionamiento, políticas y planes a las necesidades y capacidades de todas las personas. 
Ya en el Plan Internacional de Viena sobre Envejecimiento, que se había adoptado en 1982 tras la Primera Asamblea Mundial, se destacaba a las personas de edad como eje central de las políticas. Entendiéndose que este concepto se circunscribía como un proceso que se da a lo largo de la vida y que, por lo tanto, la preparación para etapas posteriores debía ser parte integrante de las políticas sociales.

Se entendió, de esta forma, que todas las personas, independientemente de la edad, podían contribuir y ser objeto de derechos, como se establece en el Artículo 1 de la Declaración Política emanada de la Asamblea Mundial:

Nosotros los representantes de los Gobiernos reunidos en la Segunda Asamblea Mundial sobre el Envejecimiento, celebrada en Madrid, hemos decidido adoptar un Plan de Acción Internacional sobre el Envejecimiento para responder a las oportunidades que ofrece y los retos que plantea el envejecimiento de la población en el siglo XXI y para promover el desarrollo de una sociedad para todas las edades. (Organización Naciones Unidas, 2002, p. 1)

De ahí que la misma Asamblea en su artículo $16^{\circ}$ reconociera la necesidad de fortalecer las relaciones intergeneracionales como fundamental para fortalecer las relaciones solidarias entre generaciones. Entendiéndose por relaciones intergeneracionales la interacción entre personas de diversas generaciones: niñez, jóvenes, personas adultas y adultas mayores. Interacción que, según el Plan de Madrid, debía ser de beneficio para todos los individuos sin importar la edad.

Históricamente, los programas intergeneracionales hicieron su aparición entre los 1960 y 1970 en Estados Unidos, cuando se descubrió que a raíz de la separación geográfica entre las personas jóvenes y las personas mayores se estaba ocasionando el aislamiento y estaban brotando percepciones estereotipadas sobre la vejez. A partir de los años 90 empieza a desarrollarse en Europa el concepto y se amplía, enfocándose en la construcción de una sociedad para todas las edades. De esta forma, en España, a partir del 2000, se dan los primeros pasos en este tipo de programas con una visión más inclusiva y con miras a impulsar un envejecimiento activo. Todo ello venía a responder al llamado que hacían las Naciones Unidas de impulsar las prácticas intergeneracionales como un método para promover la inclusión social (Asociación para el aprendizaje permanente y la participación social de las personas mayores, s. f.). 
Las relaciones intergeneracionales debían, por tanto, ocupar un espacio en las políticas públicas para garantizar dentro del contexto del envejecimiento una sociedad para todas las edades.

De ahí que La Red Intergubernamental Iberoamericana de Cooperación Técnica y el IMSERSO destacaron la importancia de fomentar las relaciones intergeneracionales como elemento fundamental para prevenir la dependencia y fomentar un envejecimiento activo y, así, poder contribuir a mejorar la calidad de vida de las personas adultas mayores (Relaciones intergeneracionales, 2009).

El Plan de Acción Internacional, en su recomendación 9a, reconocía como indispensable integrar el proceso del envejecimiento mundial en el proceso de desarrollo. En consecuencia, las políticas sobre el envejecimiento debían ser examinadas cuidadosamente desde una perspectiva de desarrollo que incluyera el hecho de una mayor duración de la vida y con un punto de vista que abarcara toda la sociedad, tomando en cuenta las recientes iniciativas mundiales y los principios orientadores establecidos en importantes conferencias y reuniones en la cumbre de las Naciones Unidas (ONU, 2002).

En todo el Plan de Acción Internacional sobre el Envejecimiento, del 2002, se planteaban varios temas centrales que estaban vinculados a esas metas, objetivos y compromisos, entre ellos destacaba la plena realización de todos los derechos humanos y libertades fundamentales.

Desde esa perspectiva, la recomendación $13^{\mathrm{a}}$ apuntaba hacia la promoción y protección de todos los derechos humanos y libertades fundamentales, incluido el derecho al desarrollo, como hechos relevantes para la creación de una sociedad incluyente para todas las edades, en la que las personas de edad participaran plenamente, sin discriminación y en condiciones de igualdad.

La lucha contra la discriminación por motivos de edad y la promoción de la dignidad de las personas de edad se tornaba fundamental para asegurar el respeto que estas personas merecen. La promoción y protección de todos los derechos humanos y libertades fundamentales eran importantes para lograr una sociedad para todas las edades. Para ello, la relación mutua entre las generaciones debía cultivarse, acentuarse y alentarse mediante un diálogo amplio y eficaz, planteaba la carta. 
De ahí que en el Artículo 16 de la Declaración Política de la Segunda Asamblea Mundial sobre el Envejecimiento, organizada por las Naciones Unidas (2002), se destacara, como eje fundamental, "reconocer la necesidad de fortalecer la solidaridad entre las generaciones y las asociaciones intergeneracionales, teniendo presentes las necesidades particulares de los mayores y de los más jóvenes y de alentar las relaciones solidarias entre generaciones" (ONU, 2002, p. 6).

Los programas intergeneracionales se constituyeron, así, en instrumentos para fortalecer relaciones solidarias entre las generaciones, y como dice VenturaMerkel y Lidoff (citados en Newman, S. y Sánchez, M., 2007) "a través de las cuales era posible compartir habilidades, conocimientos y experiencias entre jóvenes y mayores" (p. 38).

La experiencia que se desarrolló a lo largo de la década en la Universidad Nacional consistía en un acercamiento de las personas más jóvenes con las personas adultas mayores, que como lo evidencian los testimonios, se puso de manifiesto que el aprendizaje era para ambas generaciones. Los sujetos jóvenes, en un inicio, pensaban que todo su aporte iba a salir de ellos, pero se dieron cuenta que dichas personas mayores también tenían mucho por dar y que en muchas ocasiones tenían todas las facultades para hacerlo. Hecho que, de por sí, desmitificaba el estereotipo de ver la vejez como una etapa carente de capacidades y conocimientos. El intercambio era una oportunidad de crecimiento personal para ambas partes.

Tener una visión integrada por diferentes generaciones haría más factible alcanzar la meta de una sociedad para todas las edades, donde todas las personas tuvieran los mismos derechos y oportunidades sin importar la edad; teniendo presente que las relaciones entre generaciones serían beneficiosas en la medida en que fueran una oportunidad para erradicar cualquier mito de superioridad por razón de edad.

Tanto en el Plan de Acción de Viena como el Plan de Acción Internacional de Madrid sobre el Envejecimiento reconocían: "La solidaridad entre las generaciones como fundamental para el logro de una sociedad para todas las edades" (ONU, 2002, p. 19).

Asimismo, tanto la Declaración de Brasilia, del 2007, como la Carta de San José sobre los derechos de las personas mayores de América Latina y el Caribe (ALC), aprobada en la III Conferencia Regional Intergubernamental sobre Envejecimiento 
en ALC del 2012, destacaban como temas centrales la solidaridad y una sociedad para todas las edades.

Dada la trascendencia de los intercambios entre generaciones, la mayoría de países incluyeron en sus legislaciones normativas que amparaban este tipo de iniciativas. Así, por ejemplo, en Costa Rica, la Ley Integral de la Persona Adulta Mayor, Ley 7935, en su artículo 22, destacaba que este tipo de actividades debía contar con el apoyo de organizaciones tanto gubernamentales como no gubernamentales. En su decreto ejecutivo 32062, del 27 de setiembre del 2004, en homenaje a estas personas, octubre fue declarado como mes para celebrar a la persona adulta mayor, para que en lugares públicos y privados se celebraran encuentros entre generaciones que estimularan las cercanías entre personas jóvenes y personas adultas mayores.

Desde antes que a nivel internacional o nacional se estableciera como ley, ya en la Universidad Nacional de Costa Rica se estaban dando los primeros pasos de intergeneracionalidad, con miras a una sociedad más inclusiva, donde protagonistas del programa intergeneracional eran jóvenes, niños y niñas, personas adultas y personas adultas mayores.

\section{Contextualización}

El envejecimiento demográfico, definido como el aumento de personas mayores de 65 años, es un proceso que se ha venido incrementando en los últimos años, sobre todo con mayor magnitud en los países en vías de desarrollo. Si bien ha sido uno de los mayores logros de la humanidad, plantea desafíos y oportunidades en la medida que aumenta la demanda de los derechos humanos.

Los acelerados cambios demográficos también han impactado a la sociedad costarricense; en los últimos 40 años, Costa Rica venía experimentado un proceso de transición demográfica que provocaba una modificación en la estructura de edades de la población. Así, por ejemplo, mientras que para 1970 la población costarricense mayor de 65 años era de 66 mil personas, ya para 2008 había 278 mil personas adultas mayores (un 6\%) y se preveía que para 2035 la población mayor alcanzara el $15.9 \%$ (CONAPAM, 2013).

Dentro de los factores que explican estos cambios demográficos cabe destacar la disminución en las tasas de fecundidad y mortalidad, lo que le ha permitido a Costa Rica ocupar el segundo lugar de toda América. Se ha experimentado 
un significativo aumento en la esperanza de vida; según estudios realizados por CONAPAM (2013): "mientras en 1950 el promedio para hombres era de 57.7 años, para 1980 era de 74.7 y en 2010 de 79.3 años. Este fenómeno ha hecho que cada vez haya más personas adultas mayores y que vivan más años” (p. 4).

El envejecimiento demográfico implica grandes retos y transformaciones en las estructuras sociales, económicas y culturales. Estos cambios demográficos contemplan, a la vez, la necesidad de una adecuada preparación, donde se garanticen mejores condiciones para una sociedad envejecida. Es dentro de ese contexto que cobra vigencia la implementación de acciones con miras a erradicar la exclusión, la violencia y el maltrato, que son, en gran medida, producto de mitos y estereotipos que se perpetúan y mantienen de generación en generación a raíz del distanciamiento intergeneracional.

\section{Marco teórico}

La ruta hacia una sociedad para todas las edades implica erradicar prejuicios, mitos y estereotipos sobre la vejez y el envejecimiento que acentúan la discriminación hacia las personas de edad. Y para ello se requiere establecer lazos de solidaridad entre las personas adultas mayores y otras generaciones. Es en ese contexto que adquiere sentido el tema de los programas intergeneracionales. Así fue, por ejemplo, en el caso de los Estados Unidos, donde estos programas surgieron con la finalidad de reducir el enfrentamiento entre diversas generaciones.

Los mitos y estereotipos son creencias generalizadas a través de generaciones, que se perpetúan mediante la socialización. Dentro de los mitos y estereotipos más comunes en Costa Rica está el viejísimo, que consiste en atribuirle a la edad rasgos negativos, lo que viene a acentuar la diferenciación entre las personas mayores y el resto de la población, con el inconveniente de que se favorece la discriminación hacia las primeras (CONAPAM, 2013).

Una de las formas como se puede ver expresado ese mito es cuando las personas mayores son relegadas de la sociedad o recluidas en un hogar de ancianos, aun teniendo los recursos familiares y económicos para seguir viviendo en su casa, o en ocasiones se les abandona (CONAPAM, 2013).

Este concepto de abandono hacia las personas adultas mayores el CONAPAM lo complementa en su percepción de lo ya planteado por la OPS en el año 2004, 
respecto a que las cifras de personas institucionalizadas eran comparativamente mayores de aquellas que no lo estaban (CONAPAM, 2013).

Otros mitos como el asociar vejez con enfermedad, deterioro intelectual, pérdida de autonomía e improductividad son producto de prejuicios que perciben la vejez como una etapa decadente, y que, a su vez, vienen a acentuar la discriminación, el abuso y el maltrato a la persona adulta mayor, conductas violatorias de derechos humanos.

Para reforzar este tema, Estrada (citado por CONAPAM, 2007) indica:

Es recurrente la idea de que las personas adultas mayores no cuentan con un rol en la sociedad pues históricamente el envejecimiento se ha visto como un proceso de deterioro creciente, y por ello los estereotipos se han generalizado hacia valoraciones negativas hacia la vejez. (p. 2)

La creencia de que existe una superioridad por razón de edad ha llevado a fomentar una serie de mitos y estereotipos que lo que han hecho ha sido marginar y excluir a un grupo de la población que tiene los mismos derechos y oportunidades que el resto.

Erradicar la perpetuación de los mitos y estereotipos por razón de edad es fundamental, porque estos afectan la percepción que al respecto tienen los diferentes grupos poblacionales que se relacionan con las personas adultas mayores y fomentan la violencia y el maltrato. $\mathrm{Y}$ esto será posible en la medida que se intensifiquen los lazos intergeneracionales.

\section{La experiencia}

La experiencia en la Universidad Nacional, a través del proyecto "Generación Dorada", adscrito al Programa de Atención Integral, vino a responder a ese ideal de generar condiciones para estrechar lazos entre generaciones. Durante toda una década de vigencia, el proyecto logró hacer un trabajo articulado entre niñez, jóvenes, personas adultas y adultas mayores, conscientes de que el acercamiento intergeneracional permitiría reducir la brecha intergeneracional y promover el respeto por las personas de edad.

De esta forma, como parte de las prácticas intergeneracionales, se promovieron las visitas de jóvenes a residencias de personas mayores, tanto hogares de ancianos y centros diurnos, como centros penales en los cuales se promovieron 
actividades recreativas y de ocio, además de la colaboración para personas con ciertas limitaciones.

A través de las diferentes actividades que se realizaron, fue posible identificar muchas anomalías en la forma de relacionarse de la niñez y la juventud con personas adultas mayores. Una de esas fue el poco contacto que mantenían entre unos grupos y otros, como lo expresan sus propios testimonios:

Al principio no me sentía cómoda porque nunca me había relacionado con adultos mayores. Luego llegó el bingo y uno de los adultos mayores con quien más estuve hablando Víctor se puso muy alegre cuando ganó (Alpízar, 2002, párr. 4).

Aparte de entrar en conversación con ellos, ayudamos a la cocinera a servir la comida; donde nos dimos cuenta de que a cada uno se le sirve diferente: "no todos comen la misma cantidad ni las mismas cosas, sobre todo en el caso de los diabéticos" (Alpízar, 2002, parr. 5).

Esa falta de interacción con las personas mayores las hacía sentirse incompetentes para socializar con estas personas: Cuando llegué al asilo me sentí mal al ver las condiciones físicas en las que se encontraban estas personas, y la verdad no sabía qué hacer ni qué decir, pero después empecé a hablar con un señor que me hizo sentir bien, él me contó experiencias de su vida (Alpízar, 2002, párr. 3).

En realidad, creo que, como le sucedió a todos los compañeros, al principio me sentí realmente incómoda, tanto por el estado en que estaban los ancianitos como por mí misma, ya que no sabía qué hacer o decir para iniciar el diálogo (Alpízar, 2002, párr. 4).

El prejuicio del viejísimo se hace manifiesto aquí, ya que es un prejuicio que provoca que la vejez sea considerada como algo ajeno, sobre todo en una sociedad donde hay una exaltación a lo que tiene que ver con juventud al idealizarse por sus capacidades físicas y mentales. En esa dirección hay otro mito que salta a la vista: el de asociar vejez con improductividad, sobre todo si se asocia vejez con una etapa de decadencia física y mental.

$\mathrm{Y}$ es ese poco contacto con personas mayores lo que hace que estas sean desvalorizadas, sobre todo por generaciones más jóvenes: 
Al principio me sentí un poco incómodo al ver el estado en el que se encontraban los adultos mayores. Después de un rato me sentí bien ya que comencé a hablar con un señor muy simpático llamado Antonio, al principio me costó hablar con él debido a que tenía un problema en los oídos y le era difícil escucharme; después de conversar con él me di cuenta de la importancia del adulto mayor en la sociedad, por sus consejos (Alpízar, 2002, párr. 1).

Es hasta que los jóvenes entran en contacto con estas personas que descubren el valor que tienen las personas mayores en la sociedad, llegando a reconocer lo mucho que ellos pueden aportar: Me di cuenta que los ancianitos tienen mucho que dar, y que la mayoría de ellos conservan el sentido del humor (Alpízar, 2002, párr. 1).

Con respecto al baile me hizo gracia porque yo esperaba enseñar y más bien fue a la inversa, los aprendices éramos nosotros (Alpízar, 2002, párr. 9).

Mediante estas experiencias, los jóvenes no solo reconocían lo mucho que las personas mayores pueden aportar a la sociedad, sino que también rompían con el mito de que las personas por ser de edad mayor son personas amargadas, al reconocer su afecto y simpatía: Al final del día, después de ayudarlos a jugar bingo y de conversar ellos se despidieron con afecto y dulzura (Alpízar, 2002, párr. 1).

Para mí fue hermoso ver la sonrisa que cada uno tenía en su rostro, la forma como agradecían que estuviéramos ahí, sus bendiciones y consejos son una experiencia realmente inolvidable (Alpízar, 2002, párr. 1).

Inclusive llegaron a reconocer que el compartir con esas personas había sido una de las mejores experiencias, ya que les había enseñado que la edad no importa para ser alegres y poder disfrutar cada día la vida (Alpízar, 2002, párr. 6).

A través de estas actividades muchos mitos salieron a relucir, como el de asociar vejez con discapacidad:

Cuando quise ayudar a un señor a cambiarse del sillón a la silla de ruedas me regaño y me dijo que no le tocara la silla, que él podía solo, y lo logró. 
Con eso aprendí que muchos todavía pueden valerse por sí mismos y que tienen mucho espíritu y fuerza de voluntad (Alpízar, 2002).

El trato discriminado que muchas personas adultas reciben ha llegado a afectar su autoestima, sobre todo porque muchas personas no les prestan la debida atención a sus propias historias. Pero conforme se da el acercamiento entre ellas y jóvenes se empiezan a sentir valorados:

Pienso que este día fue muy agradable para ellos porque se les llevó música, comida y además de eso todos los estudiantes que ahí se encontraban tratamos de hacerle el rato lo más ameno posible, todos les brindamos atención en el momento que nos contaban sus historias y nos hacían reír con sus experiencias. Creo que actividades como estas les hace muy bien, pues de esta manera todavía se sienten queridos y no se sienten olvidados o rechazados (Alpízar, 2002, párr. 3).

El acercamiento hacía que las percepciones equivocadas sobre las personas adultas mayores fueran modificándose:

Pero todo fue cambiando a medida que empecé a hablar con ellos, con el primero que conversé fue con Salomón, el cual me contó su vida y la de los compañeros; él fue el que me puso al tanto del estado de algunos viejitos, de los cuales la mayoría lo único que necesitan es que alguien los escuche y los haga sentir valiosos (Alpízar, 2002, párr. 4).

Estas formas de convivencia, de diálogos intergeneracionales hacen que los jóvenes logren crear conciencia social y aprender a valorar a las personas mayores, como ellos mismos lo expresaban. Inclusive reconocían que:

Este proyecto les permitía reflexionar sobre el futuro que les esperaba a todos como adultos mayores, y que los motivaba a valorar más a los ancianos, los humanizó y logró acercarlos a sus sentimientos y emociones (Alpízar, 2002, párr. 2).

Inclusive los mismos grupos jóvenes lograban reconocer que esta población era marginada e ignorada, y se olvidaba que había sido el pilar de la sociedad. De ahí que consideraran necesario concientizar sobre el trato que la sociedad comúnmente da a las personas ancianas y poder así aprender de los errores que los mismos familiares cometen al separarles de sus hogares. 
No cabe duda, que el poco contacto que generalmente ha predominado entre la juventud y las personas adultas mayores ha potenciado las conductas de agresión, maltrato y hasta de exclusión, sobre todo en una sociedad donde hay una desvalorización social de la vejez por considerarse una etapa de pérdida de facultades físicas y emocionales. Como bien lo demuestran los grupos de jóvenes al sentirse impresionados por el desenvolviendo y las muestras de alegría mostradas por las personas adultas mayores.

En medio de estas circunstancias, las personas participantes de este proyecto apostaban al ideal de que: los jóvenes de hoy en día supieran apreciar el verdadero valor de los adultos mayores, como personas maravillosas de la sociedad.

El acercamiento intergeneracional se vio complementado mediante las actividades que se generaron como parte de la celebración del mes de la persona adulta mayor, que se empezaron a realizar en octubre dentro del campus universitario; desde la cual se fomentó la participación de personas de todas las edades en actos culturales y artísticos. De esta forma era evidente que quienes se beneficiaban del programa intergeneracional eran todas las personas participantes y la sociedad en general.

En la directriz $19^{\mathrm{a}}$ de La Declaración Política de la Segunda Asamblea Mundial sobre el Envejecimiento, se enfatiza que para lograr que las personas de edad tengan la oportunidad de seguir contribuyendo a la sociedad, es necesario eliminar todos los factores excluyentes o discriminatorios en contra de ellas, ya que su contribución social y económica debe de ir más allá de sus actividades económicas, pues con frecuencia desempeñan funciones cruciales en la familia y en la comunidad (ONU, 2002).

Como bien lo reconocían un número de jóvenes, quienes al principio dudaban de que fueran a aprender algo de las personas mayores les tomó de sorpresa, ya que terminaron reconociendo que ellos o ellas tenían mucho que aprender de las personas adultas mayores. Esto daba al traste con el estereotipo de que las personas adultas mayores son improductivas.

Muchos de los valiosos aportes de las personas adultas mayores no se miden en términos económicos, como en el caso de los cuidados prestados a miembros de la familia, el trabajo productivo de subsistencia, el mantenimiento de los hogares y la realización de actividades voluntarias en la comunidad. Además, esas funciones contribuyen a la preparación de la fuerza de trabajo futura. Es necesario reconocer todas esas contribuciones, incluidas las del trabajo no remunerado que 
realizan en todos los sectores las personas de todas las edades, y en particular las mujeres, destacaba la Segunda Asamblea Mundial sobre el Envejecimiento.

El proyecto también involucraba entre sus actividades el desarrollo de una serie de temas que facilitaran a las personas de todas las edades, el irse preparando en su proceso de envejecimiento.

El acelerado envejecimiento implicaba la necesaria definición de nuevas políticas tendientes a favorecer un envejecimiento con calidad, que influyera en la forma de enfrentar la vejez de manera sana y activa. Para lo cual se requería, como lo apuntaba el Plan de Acción Internacional de envejecimiento 2002, de cambios de actitudes, de políticas y de prácticas en los diferentes grupos etarios, con el fin de preparar a toda la población para las etapas del envejecimiento.

Para ello era fundamental la promoción y protección de los derechos humanos, para que existiera una sociedad que incluyera a todas las edades, que facilitara la participación de las personas mayores sin discriminación alguna. Involucrar diferentes grupos etarios hacía posible potenciar la erradicación de prejuicios y valoraciones negativas con respecto a la vejez y el envejecimiento.

Con ese propósito, se desarrolló un proyecto de capacitaciones que permitía no solo un mayor acercamiento entre los diferentes grupos etéreos, sino también un acercamiento de las personas adultas mayores a la Universidad. Las personas de edad de hace más de una década les costaba asimilar la idea de la oportunidad que tenían para incorporarse a una institución de educación superior que les abría las puertas. Tanto ellas como las poblaciones más jóvenes debían de erradicar ciertos mitos; unas, para poder involucrarse con toda libertad y otras para erradicar toda actitud excluyente hacia las personas adultas mayores.

Las experiencias para fortalecer esas relaciones intergeneracionales incluían: visitas a hogares y centros diurnos, visitas a centros penales de personas adultas mayores, realización de charlas sobre temáticas afines a diferentes edades, visitas a centros educativos, creación de la celebración del mes de la persona adulta mayor, y supervisión de servicio del transporte público, en cuya ejecución colaboraban estudiantes del curso de Derechos Humanos del Centro de Estudios Generales de la Universidad Nacional.

Durante toda la década que estuvo vigente este proyecto se visitaron más de 20 centros de atención a personas adultas mayores. Entre ellos cabe destacar los siguientes: 
Asociación Pro-Atención Integral, Barva, cuya población estaba constituida por personas adultas mayores con limitaciones físicas y mentales. Esta experiencia fue impactante, ya que estas personas requerían de mayor apoyo y cariño que sus familiares no les podían dar por tener que trabajar. Ante lo cual surgió la interrogante entre estudiantes: "¿cómo atender a los adultos mayores si los tiempos modernos obligan a salir a trabajar, teniendo que dejar al cuidado ajeno hasta a los mismos hijos?" (Alpízar, 2001, párr. 5).

En Hogares como Madre Berta Acuña Ruiz, San Pablo de Heredia, la interacción de estudiantes estuvo enfocada a escuchar a las personas ancianas, quienes estaban ansiosas de contar su vida. Al respecto los grupos de estudiantes manifestaban:

Es evidente el deseo que tienen de que alguien les preste atención, ya que la mayoría que ingresó a ese hogar fueron llevados mediante engaños, al decirles que solo estarían ahí por un poco de tiempo y, sin embargo, sus familiares nunca volvieron a visitarlos lo que les llena de tristeza, soledad y amargura. (Alpízar, 2001, párr.5).

Ante expresiones como estas, el estudiantado manifestaba que, pese a que en un principio estaba desmotivado para visitar estos centros, luego reconocieron que era tan significativo por el vacío que llenaban en estas personas. Mediante estas visitas lograban adquirir conciencia respecto de su propia vejez, la cual no deseaban pasarla recluidos en un hogar de ancianos.

En el Hogar de Ancianos Rodrigo Crespo, Alajuela, los grupos de jóvenes interactuaban con las personas mayores mediante juegos como el bingo y, en el caso de no videntes u otra limitación física, lo hacían en su nombre. También mostraron solidaridad compartiendo alimentos que lograban obtener por donación de algunas casas comerciales.

En otros hogares, como el Hogar Ofelia Carvajal de Desamparados, Misioneras de la Caridad en Coronado, Hogar Carlos Manuel Ulloa, el Hogar de Ancianos de Palmares, Hogar Manos de Jesús en Cartago, Centro Diurno de Tucurrique, Asociación Esperanza Cristiana para el Anciano en Guadalupe, Hogar Carlos María Ulloa, San Francisco de Asís, Hogar Diurno de Santa Ana, el Hogar para Ancianos Alfredo y Delia González Flores, se hizo trabajo social, al contribuir a facilitarles a las personas institucionalizadas ayuda para alimentarse, para 
cuidados personales. También fue una oportunidad para escucharles, y realizar actividades culturales.

Otro lugar visitado fue El Hogar de Ancianos Albernia, San Isidro de Heredia, cuyos residentes en esa ocasión eran personas con limitaciones físicas y mentales. Entre las actividades que se desarrollaron en este lugar, con el apoyo de estudiantes, estuvo también la capacitación al personal con respecto al trato y cuidados a personas adultas mayores y la socialización con ellas.

Las personas adultas mayores han sido objeto de tanta discriminación que han creído que por su edad ya no tienen derecho ni a jugar. Como lo expresaban algunas, quienes se resistían a involucrase en algunos juegos como el de la papa caliente por considerarse muy viejas y que ese tipo de juegos era para niños. Sin embargo, luego de un rato, cambió su forma de pensar y se integraron al juego. Percepción semejante tenía un adulto mayor, quien se negaba a comer confites, ya que consideraba que eran solo para niños. Gracias al trabajo desarrollado por los grupos de jóvenes, esas percepciones variaron considerablemente en estas personas, quienes posteriormente se integraban a las actividades.

Las oportunidades que se abren a las personas mayores en la convivencia con personas jóvenes son grandes, ya que muchas aprovechan el espacio para contar historias de vida, mostrando gran interés por que se les escuche sobre todo en los casos en los cuales las personas habían ingresado bajo el engaño, como sucedía en algunos centros visitados.

Los jóvenes descubrían por sus propios medios las necesidades afectivas que tenían muchas personas mayores:

Es evidente el deseo que tienen de que alguien les preste atención y les escuchen, ya que la mayoría había ingresado bajo engaño, al decirles que estarían por poco tiempo, sin embargo, sus familiares no regresaron, lo que les llena de tristeza, soledad y amargura. (Alpízar, 2001)

Las formas como los jóvenes mostraron solidaridad hacia las personas adultas mayores fueron variadas, dependiendo de la situación en la que se encontraran estas personas. Así, por ejemplo, en el caso del Albergue de Ancianos Banquete Celestial, se gestionaron alimentos y colaboraban directamente con el cuidado personal haciendo manicura, pedicura, corte de cabello, entre otros. 
Por la naturaleza de las personas albergadas, que en su mayoría eran personas mayores en condición de indigencia y por carecer de financiamiento fijo en ese momento, se gestionó el apoyo de un profesional en terapia física, quien brindaba acondicionamiento físico para personas mayores, inclusive para las encamadas. Como parte del trabajo solidario, se logró establecer un convenio con la Clínica Jorge Volio, de San Joaquín de Flores, para que se encargara de visitar semanalmente a las personas mayores de este albergue.

También se les impartieron talleres para capacitar al personal cuidador en la atención de personas mayores con alguna demencia y en el tema de los derechos.

Mediante este proyecto se logró reflexionar sobre la condición de las personas adultas mayores, respecto de la necesidad de mejorar el trato, tanto por parte de las familias como de los centros de atención y de la sociedad en general. Se inculcaron valores de respeto, estima y se despertó en el estudiantado una mayor sensibilización y solidaridad para con una población en condición de vulnerabilidad.

Con el propósito de alcanzar la mayor cantidad de familias se visitaron centros diurnos, como La Casa de los Abuelos, en Cubujuquí de Heredia, la Casa Diurna del Adulto Mayor Esperanza del Zurquí, en los Ángeles de Santo Domingo de Heredia, Hogar de Ancianos Jafeth Jiménez Morales en Grecia, Centro Diurno de Los Lagos, Centro de Atención Integral de Ancianos Francisca Valerio, San Rafael de Heredia. Al igual que otros centros visitados, los grupos de jóvenes aquí lograron tener un acercamiento con esta población a través de actividades recreativas como bailes, sesiones de chistes y actividades de acondicionamiento físico. Al mismo tiempo que se promovieron las capacitaciones del personal cuidador orientadas a mejorar el trato hacia las personas adultas mayores.

Para la mayoría de estudiantes era la primera vez que visitaba un centro de ancianos, y les era de gran impacto, sobre todo cuando encontraron casos de personas mayores que estaban en esos lugares producto del engaño que les habían hecho sus propias familias, y que, pese a tener varios años de estar en ese lugar, aún seguían esperando a sus seres queridos.

Para gran cantidad de estudiantes era una situación incómoda, ya que no sabían cómo reaccionar, ni cómo comportarse ante esta población desprotegida por su descendencia más cercana, y aún más incompetentes se sentían ante "el llanto desconsolado de los abuelitos, ante un ambiente de tristeza y soledad; al encontrar que los ancianos esperan sin cesar la visita de un familiar". 
Al acercarse a los hogares de ancianos los grupos de estudiantes manifestaron encontrarse con corazones vacíos, llenos de soledad, limitados a estar sentados en un rincón. Percibir ciertos tratos grotescos y expresiones burlescas de parte del personal de algunos centros de atención a personas mayores les causó mucho impacto. Tal fue el caso en una de las ocasiones que ellos promovieron una actividad recreativa y las personas reaccionaron muy emotivas, ante lo cual un funcionario manifestó: "Ahora sí, ya no les duele nada como para estar quejándose” (Alpízar, 2001, párr. 7).

De hecho, estas experiencias dejaban una huella en los grupos de jóvenes que tenían la oportunidad de compartir con esta población, acerca de la necesidad de sensibilizar más a los cuidadores y cuidadoras en el trato a las personas adultas mayores. En el acercamiento que empezaron a tener estudiantes con personas de algunos centros institucionalizados, les impactaba la indiferencia, las palabras descalificantes, por parte de algunos cuidadores. Por lo tanto, llegaron a reconocer la necesidad de promover mejorar el trato hacia la persona adulta mayor, fomentar el respeto y valoración hacia estas personas y reducir los índices de agresión hacia ellas.

Por el poco conocimiento y poco o ningún contacto que los grupos de estudiantes tenían con las personas adultas mayores, se sentían incompetentes para acerarse a ellas y poder brindar ayuda. Fue frecuente escuchar el temor que persistía en ellos para hacer cosas como darles de comer o actuar ante el llanto desconsolado de una abuelita.

De ahí que consideraban que más necesitados que los ancianos y ancianas estaban ellos de aprender a convivir con una realidad que para la mayoría era desconocida. Así, por ejemplo, ante un anciano que con tristeza vociferaba durante la visita: "los perdono a todos" ellos llegaron a la conclusión de que posiblemente se refería a sus familiares y a la sociedad como un todo (Alpízar, 2001, párr. 7).

Estas experiencias les inducían a reflexionar sobre la condición de sus padres, madres y de ellos o ellas mismas como personas adultas mayores. Por lo cual recomendaban que este tipo de trabajos fuera requisito para toda la ciudadanía, dada la gran necesidad afectiva que hay en las personas institucionalizadas y el gran analfabetismo sobre el tema.

Fue tal el impacto de las expresiones citadas, que los grupos de estudiantes se dispusieron a realizar más visitas de esta naturaleza en centros para personas 
mayores, al punto de que recomendaron que este tipo de experiencias debían de ser vividas por adolescentes, a fin de que se fomentara un mayor respeto por la persona adulta mayor.

La soledad y el abandono era tan evidente en muchos de estos lugares que era común escuchar frases como: “¿Cuándo regresan?” o "Gracias por venir”, "No olviden el camino", lo que inclusive despertaba emotividad en el estudiantado, quienes desconocían que fuera tan significativas las visitas para estas personas.

De ahí que consideraran la experiencia era muy gratificante: "consideramos tan valioso este proyecto cuando no son grandes pensantes, libros gruesos, exposiciones aburridas, exámenes tediosos, sino nuestra experiencia lo que nos enseña la importancia de ver lo que estamos haciendo con nuestros viejitos..." (Alpízar, 2002, párr. 7).

Al detectar la carencia de cariño que tenían de parte de sus hijos e hijas, el abandono de que eran objeto, el estudiantado se cuestionó el hecho de cómo enfrentar esta situación ante la necesidad de salir a trabajar.

Ante estas situaciones, se mostraron a favor de que el sistema educativo incentivara más a la niñez y a la juventud a compartir con las personas adultas mayores a fin de evitar tanta soledad en las personas institucionalizadas.

Si bien las personas que estaban en centros o casas de cuido presentaban síntomas de soledad y abandono, existía una población que ha estado doblemente marginada; era la población adulta mayor privada de libertad. De ahí que a partir del 2001 se dieran los primeros pasos de acercamiento intergeneracional con estas personas. Durante la convivencia se ejecutaron una serie de actividades recreativas y culturales a través de las cuales se estrechaban lazos de solidaridad con personas doblemente marginadas. Y por el poco conocimiento de esta realidad se consideró importante desarrollar un tema como el de la problemática física y psicológica de las personas adultas mayores privadas de libertad, cuyo facilitador fue el entonces director del Centro Institucional de Adulto Mayor de La Reforma.

Trabajar con personas adultas mayores privadas de libertad fue una experiencia muy significativa, por la condición de privados de libertad y, consecuentemente, por el impacto que ocasionaba ver a estas personitas en esas condiciones; las visitas a dichos centros se realizaban con estudiantes de los cursos de Derechos 
Humanos, quienes mostraban mucho interés por contribuir a paliar la soledad de dichas personas.

Nos propusimos acercarnos no solamente a la cárcel de hombres, sino también incursionar en la cárcel de mujeres. Detectamos que había un centro dedicado a adultos mayores hombres privados de libertad, pero que, para el caso de las mujeres mayores, ellas debían compartir la condena con personas más jóvenes, con los inconvenientes que ello significaba. Varias razones podían justificar la ausencia de un centro penal exclusivo para adultas mayores, como el hecho de que la población femenina adulta mayor era más escasa. Pero lo cierto es que no se descarta el factor de género que podía estar incidiendo en esta discriminación, que ya de por sí tenía sus implicaciones negativas para esa población.

En ese sentido, las mujeres adultas mayores no recibían ningún trato preferencial por razón de edad. La alimentación, los dormitorios, higiene y cuidados médicos eran iguales para todas, pasando por alto el trato preferencial que de por sí merece la persona adulta mayor.

A partir de ese año también se buscó tener un acercamiento con personas adultas mayores que permanecían en sus domicilios, con poca o ninguna compañía. El propósito era no solo ofrecerles compañía sino también hacer de su conocimiento sus derechos humanos, facilitarles algunos servicios, valorar la compañía de los abuelos y abuelas e identificar algunas problemáticas para su posible solución. El trabajo era realizado por estudiantes de la Universidad Nacional, y se enfocó no solo en las personas vecinas de este centro educativo, sino también de sus domicilios. De esta forma se generaba un enriquecimiento mutuo, ya que se despertaba un mayor interés en estudiantes por las personas adultas mayores, se fomentaba un mejor trato, fortaleciéndose así la labor de sensibilización y respeto por las personas adultas mayores.

En el afán de impulsar un acercamiento intergeneracional, se debía incorporar la participación de la niñez y adolescencia con el fin de sensibilizarles y fomentar una relación respetuosa hacia las personas adultas mayores.

Con la experiencia que había vivido el estudiantado, se había despertado la preocupación por el hecho de que el sistema educativo no incentivara en esos tiempos a los niños, niñas y jóvenes a compartir más con las personas adultas mayores; ya que la experiencia vivida había sido tan valiosa que la consideraban, no solo un requisito de estudios sino una necesidad para continuar viviendo, ya 
que les tocaba el corazón la frase de despedida que por lo general los ancianos expresaban: "no olviden el camino" (Alpízar, 2001, párr. 1).

Esto facilitó que los grupos de jóvenes se dispusieran a colaborar con el proyecto de acercamiento a centros educativos a fin de sensibilizar a la población más joven e infantil. De esta forma se procedió a visitar algunos centros educativos, como la Escuela José Ezequiel González Vindas, de San Pablo de Heredia, en cuyo lugar se trató el tema de los derechos humanos. Mediante las dinámicas que se desarrollaron se logró que los niños y niñas compartieran sus experiencias con los abuelitos y abuelitas, y que se concientizaran acerca del valor de estas personas, de modo que no se avergonzaran de ellas. Una de las técnicas usadas fue la de la pecera, a través de la cual tenían la posibilidad de identificar aquellos derechos humanos más violentados en las personas adultas mayores.

Otras de las instituciones visitadas fue el Instituto Educacional Bachillerato a Distancia, de Tibás. Este lugar fue seleccionado por ser un centro educativo de carácter técnico, al cual también debía de sensibilizarse para "educar la conciencia", manifestaba el estudiantado universitario. Para esta ocasión se contó con el apoyo de AGECO, con lo cual se logró, en medio de la resistencia, que estudiantes se involucraran más en comentar sus experiencias con sus abuelitos. Con este grupo se abordó el tema de los derechos humanos, y el de la agresión y el maltrato hacia las personas mayores (Alpízar, 2001, párr. 1).

Se alcanzó también a grupos organizados como los Scoutt de Santo Domingo, donde participaron niños con edades entre los 8 y 13 años. Con ellos se abordó el tema de la agresión, para lo cual se usó como referencia la noticia publicada en el periódico La Nación, donde se relataba el caso de un anciano a quien las ratas le habían comido la nariz. A pesar de haberse publicado en un medio de comunicación, era un tema desconocido para los niños, quienes se mostraron muy impactados y movidos a interesarse más por sus mayores.

Otros centros educativos visitados fueron: La Escuela San José, de San Josecito de San Rafael de Heredia, La Escuela Braulio Morales Cervantes de Heredia, el Colegio Villaseca de los Misioneros Josefinos. En Alajuela también participaron otros centros educativos como la Escuela San Agustín del Coyol de Alajuela, con quienes se trabajó el tema de los derechos humanos de las personas adultas mayores. También se trató, mediante una dramatización, el tema de la agresión a las personas adultas mayores. Se reforzó el manejo de la problemática con el 
tema de los derechos humanos. En este caso, los niños manifestaron que ya no les iban a gritar a sus abuelitos ni abuelitas, que se iban a portar bien con ellos, y, en el caso de los que estaban institucionalizados, le iban a pedir a sus padres que asumieran ellos mismos el cuido (Alpízar, 2001, párr. 1).

En la Escuela Divino Niño en San Antonio de Belén, se abordó el tema de los cuidados especiales que merecen las personas mayores y sobre los tipos de agresión a las personas mayores. La temática se desarrolló mediante dramatizaciones para reflejar las formas comunes de agresión a esta población. Con la misma temática se trabajó en el CINDEA de Santa Ana, solo que con una metodología diferente, cual fue la obra de teatro. En el centro educativo Colegio Santa Teresa, del Pacto del Jocote en Alajuela, se trató el papel de las personas adultas mayores en la sociedad, y el papel de jóvenes como futuros abuelos y abuelas.

Con las dinámicas, charlas y obras de teatro, realizadas por los grupos de estudiantes de derechos humanos se buscaba despertar, en poblaciones más juveniles, respeto hacia las personas mayores, quienes en ocasiones expresaban, que los abuelos y abuelas eran buenos por cuanto les daban plata, regalos y les cuidaban; pero en otros casos manifestaban que molestaban mucho, se entrometían, querían decidir por ellos y tratar de inculcar sus ideas. (Alpízar, 2001, párr. 3).

En Moravia se visitó el Colegio María Inmaculada y se trabajó particularmente con niños y niñas de preescolar. Mediante dinámicas especiales se inculcaron valores asociados al respeto de las personas adultas mayores.

El Liceo Experimental Bilingüe de Grecia y otros centros educativos también fueron visitados: Jardín de Niños Valle Verde de San Luis de Santo Domingo de Heredia y el Kínder de Mi Tía Panchita.

En la Escuela Cleto González Víquez de Heredia se impartieron charlas acerca de las necesidades y cuidados de la persona adulta mayor, donde sorprendentemente se escucharon comentarios bastantes negativos al manifestar que "había que tratarlos mal porque eran muy necios y no permitían hacer las cosas que ellos querían hacer". Otros, por su parte, estaban alejados de sus abuelos porque sus papás estaban enojados o inclusive porque los desconocían. Para los grupos de estudiantes, la labor desarrollada en ese centro educativo venía a concientizar a estos niños y niñas para mejorar la percepción y el trato hacia las personas adultas mayores (Alpízar, 2001, párr.1). 
Este tipo de expresiones revelan la marginación a la cual han estado expuestas las personas adultas mayores por parte de su propia familia. No dudamos lo revelador que era para los grupos universitarios de jóvenes la situación de maltrato a dicha población. Y, por lo tanto, lo oportuno que resultaba el acercamiento intergeneracional para reducir la brecha y así inculcar un mayor respeto por las personas adultas mayores.

De ahí que durante la experiencia con niños y niñas de centros educativos se provocó tal conciencia que hubo quienes inclusive llegaron a manifestar que ya no les iban a gritar más a los abuelos y abuelas, y que les iban a pedir a sus padres que les sacaran de los hogares de ancianos.

Las actividades desarrolladas en centros educativos y centros de atención a personas mayores fueron complementadas con la creación de la celebración del día de la persona adulta mayor en el mes de octubre, para el que quedó establecido el primer jueves de octubre como el día oficial. En esta fecha se empezaron a organizar encuentros intergeneracionales, y se logró así celebrar el Primer Encuentro Intergeneracional en 2000, en el cual hubo una destacada participación de niños, niñas y jóvenes de primaria y secundaria, estudiantes de universidades y personas mayores, quienes con su talento artístico lograron ponerle sabor a la actividad. En dichos encuentros intergeneracionales sobresalían coreografías, bailes típicos, juegos tradicionales, concursos de baile y canto, exposiciones de artesanía, con participación de personas de diferentes edades, incluyendo las adultas mayores.

No cabía la menor duda de que estábamos construyendo las bases para erradicar las brechas intergeneracionales, mitos y estereotipos y fomentar el respeto y el cariño por las personas de edad. Por ello se declararon estos encuentros como actos oficiales para ser celebrados anualmente en el mes de octubre.

En ellos, se contaba con la participación de la marimba de la UNA, con miembros de AGECO sus bailes folclóricos y sus artesanías. Concurrían personas adultas mayores de la comunidad nacional, centros diurnos y hogares de ancianos, incluyendo personas del Albergue Banquete Celestial, quienes en sus mayorías eran de condición de indigencia o con limitaciones físicas. La alegría que irradiaban los rostros de todas las personas mayores era impresionante, era la primera vez que la Universidad Nacional ofrecía un evento que los hacía sentirse importantes. 
También participaban estudiantes de centros educativos, como la Escuela La Puebla de Heredia y el centro Infantil de la UNA (CIUNA).

Con las actividades que se extendían durante toda la semana, se hacían exposiciones de manualidades o ferias artesanales con los clubes de AGECO; actividades recreativas como juegos de ajedrez, actividades culturales con presentación de música antigua y hasta un club literario; se propiciaba así la interacción entre estudiantes, personal funcionario y personas adultas mayores, desterrando así la idea de que los centros de educación superior eran recintos de exclusividad juvenil.

-El encuentro que se celebró en el año 2002 y al cual asistieron más de 100 personas fue considerado como pionero en el rango de las actividades intergeneracionales. Se establecía, así, la celebración oficial del día de la persona adulta mayor. Era la primera vez que este tipo de actividades se publicaban en un medio de comunicación como La Nación. En esta ocasión, aparte de los grupos que ya habían participado, también se incorporó la participación del Colegio Humanístico.

Se contó con una amena presentación de tango, concurso de coplas y, por supuesto, la marimba no podía faltar. Las personas adultas mayores empezaban a sentirse reconocidas y valoradas, y las más jóvenes empezaban a estrechar lazos con personas portadoras de sabiduría y experiencia y así poder valorar sus enseñanzas:

Una de las cosas más importantes es haber aprendido de estas personas tan especiales, pues pude darme cuenta que son seres humanos, son ejemplo para todos nosotros; siguen demostrando que son personas útiles y que tienen mucho que ofrecer a quienes le rodean; expresaban los estudiantes. (Alpízar, 2001, párr. 1)

Nos despedimos de ellos, en mi caso, y yo sé que en el caso de mis compañeros, hubo un sentimiento de satisfacción y de gran alegría por haber compartido con personas que nos dan una gran lección y enseñanza para hacer las cosas bien en la vida; nos dan testimonio de que sus errores deben servir para que aprendamos de ellos y no los cometamos... (Alpízar, 2001, párr. 1)

Con el propósito de intensificar el acercamiento intergeneracional, a partir del año 2005 se incluyó otra serie de actividades. Entre ellas se promovió un concurso de historias doradas y se procedió a homenajear a las personas centenarias. Las 
personas centenarias que participaron en esa ocasión fueron 5: 4 mujeres y un varón, con edades entre 101 y 103.

Si bien esta fue una ocasión para que estas personas escribieran su historia de vida, lo cierto es que la participación fue muy escasa. Muchos factores pudieron mediar para se diera esta situación, entre ellos el poco acceso a medios de comunicación escrita, así como el hecho de desconocer este tipo de oportunidades. Como dato curioso, también influyó en cierta medida el hecho de que no se ofreciera recompensa económica por escribir su historia, y ello se constató por las llamadas que hicieron algunas personas para consultar si pagaban por su participación. Y como no se identificaban, no se sabe con certeza si era la persona mayor o eran sus familiares los que buscaban retribución.

Pese a la poca participación que hubo de personas centenarias, y lo cual puede tener su explicación en la escasa población con esas edades, lo cierto es que era la primera oportunidad que tenían estas personas para recibir reconocimiento por razones de su edad.

Mediante todas esas estrategias desarrolladas que fomentaban el intercambio intergeneracional se estaban sentando las bases para una sociedad para todas las edades. Pero aún quedaban aspectos que no eran congruentes con esos ideales. Uno de ellos era la forma como se estaba ofreciendo un beneficio a las personas adultas mayores por razón de su edad, el cual era el transporte público subvencionado. Con el propósito de contribuir a garantizar un servicio de calidad se procedió a evaluar el servicio del transporte público, lo cual se ejecutó a partir del año 2003, un año después de haberse establecido a nivel nacional dicho servicio.

El servicio subvencionado de transporte público, para personas adultas mayores, se había implementado en Costa Rica a partir del año 2002 en el marco de la Ley Integral para la persona Adulta Mayor n. 7935, del 15 de noviembre de 1999. Se hizo una modificación al artículo 33 de la ley 7936 con el propósito de mejorar los servicios públicos para permitir un mejor tratamiento y más eficiente servicio a la comunidad de personas adultas mayores, conforme a sus necesidades reales. Según la Ley Reguladora del Transporte Remunerado de Personas en Vehículos Automotores n. 3503 de 10-5-1965 en su artículo 33, destacaba que: 
Las personas mayores de 65 años viajarían sin costo alguno en los desplazamientos que no excedieran de 25 kilómetros. En los desplazamientos mayores de 25 kilómetros y menores de 50 kilómetros, pagarían el cincuenta por ciento (50\%) del pasaje; en los desplazamientos mayores de 50 kilómetros, el costo era de setenta y cinco por ciento $(75 \%)$ del pasaje.

El artículo 3 de la ley mencionada anteriormente, sobre este particular, indica:

De previo a la exoneración del pasaje prevista en el inciso b) del artículo anterior, los usuarios deberían presentar al chofer de la unidad de transporte público, su cédula de identidad y el carné de ciudadano de oro, el cual será extendido por la Caja Costarricense de Seguro Social, a efectos de demostrar que realmente son personas mayores de 65 años. (1965)

De esta forma, en el año 2002 se establecía en nuestro país este tipo de servicio para las personas mayores.

Con el propósito de acercarnos a conocer la calidad del servicio, se visitaron algunas rutas, en las cuales se identificaron algunas anomalías, pese a que el instructivo para la entrega de tiquetes de exoneración del pago a "Ciudadanos de Oro" establecía todo un procedimiento.

Dentro de las anomalías encontradas estaban:

1. Los choferes no daban el tiempo prudencial a las personas adultas mayores para ocupar sus asientos, lo que provocaba, en muchas ocasiones, que estas personas salieran lesionadas ante la forma brusca de arrancar el autobús.

2. La clasificación de los tiquetes por colores no era la más apropiada, dado que se prestaba para confusiones y, consecuentemente, para humillaciones.

3. La obligatoriedad de presentar la cédula y carné de oro se convertía en un atraso para los usuarios y choferes en general.

4. Las empresas restringían la cantidad de tiquetes por día, lo que alteraba el servicio.

5. El uso de barras electrónicas se convertía en un obstáculo para personas ancianas con alguna limitación y hasta en un motivo de humillación.

6. El considerar que las personas que cancelaban con dinero efectivo tenían más derecho que quienes pagaban con tiquetes.

7. El poco conocimiento sobre el beneficio de la ley 7936. 
8. Los choferes expresaron molestia por la lentitud de las personas adultas mayores al pasar las barras.

9. Según manifestaban las mismas personas adultas mayores, el procedimiento para obtener los tiquetes era un poco engorroso, tanto por las humillaciones a que estaban expuestas, como por lo complicado que era el manejo de tiquetes de diversos colores. Por lo que el beneficio al principio no era tan apetecido y muchos preferían renunciar a él.

Aunque las condiciones para gozar ese derecho no estaban claramente definidas, lo cierto es que la sociedad costarricense en ese momento y los encargados de ofrecer el servicio tampoco estaban preparados para brindarlo de la mejor manera, lo que ocasionaba constantes atropellos a los usuarios y usuarias. En una evaluación general se encontraron algunas anomalías. Era evidente la necesidad de hacer campañas de sensibilización y de alfabetización, a fin de ir erradicando los prejuicios que tornaban dicho beneficio en algo hostil.

Pese a la resistencia que mostraban choferes y empresarios, lo cierto es que para las personas adultas mayores tuvo gran significado el interés mostrado por la Universidad por evaluar la calidad de servicio de transporte con la modalidad de los tiquetes.

Para realizar esta valoración sobre la calidad del servicio de transporte brindado a las personas adultas mayores con el uso de los tiquetes, se contó con la colaboración de estudiantes del curso de Derechos Humanos de la Universidad Nacional. El proceso implicó interacción con choferes de buses, entes empresarios y personas usuarias del servicio. Mediante un trabajo de observación y entrevistas, se identificaron casos de maltrato y discriminación a personas mayores por su condición de usuarias del servicio de buses. Esto implicaba la necesidad de fomentar una mayor sensibilización con la situación de vulnerabilidad de las personas adultas mayores y, por otro lado, de reconocimiento de un derecho que estaba plenamente establecido: este era el propósito de nuestra intervención.

Después de uno y varios diagnósticos hechos por diferentes instancias, y de campañas de sensibilización y de ligeros cambios en la estructura de los tiquetes, la situación ha ido mejorando, pero no lo suficiente como lo merecen las personas adultas mayores. Aún queda mucho por hacer en este campo.

No cabe duda de que esto constituía un gran paso hacia una sociedad menos discriminatoria por razón de edad y, por ende, hacia una sociedad para todas las edades. 
Posterior a esta experiencia, y con la misma visión de involucrar a los diferentes grupos etarios, se empezaron a ofrecer talleres dirigidos a personas adultas mayores, cuyo propósito era consolidar la apertura de las puertas a la educación de las personas mayores y, consecuentemente, contribuir a una mejor preparación para afrontar su propia vejez.

Durante una década, como parte de este proyecto, se trataron una serie de temas, como: andropausia y menopausia, fisioterapia, agresión a la persona adulta mayor, sexualidad, cambios físicos y emocionales después de los 40 , medicina alternativa, depresión, muerte y duelo, alimentación y salud, teatro, derechos humanos, la belleza después de los 60 , estilos de vida saludable, prevención y tratamiento de la osteoporosis, alzhéimer, las caídas, violencia intrafamiliar, enfermedades crónicas, ejercicio y calidad de vida en la persona adulta mayor, abuso y maltrato de la persona adulta mayor, síndrome de inmovilidad, ocio y recreación, sanidad interior, autoestima, farmacología, violencia patrimonial, homeopatía, mitos y estereotipos de la vejez, duelo, cuidados bucodentales, jubilación, deterioro sensorial, vejez y envejecimiento. Con su desarrollo se buscaba hacer conciencia sobre temas que hasta ese momento poco o nada se trataban.

Para incentivar un mejor aprovechamiento del tiempo libre, se impartieron talleres de arte y de cine, caricatura, y recitales de poesía y cuento. Todos fueron impartidos por profesionales, durante toda una década y en los cuales hubo concurrida asistencia de personas de todas las edades, tanto de parte de personas de la comunidad como personal y estudiantes de la universidad.

Mediante la puesta de marcha de todo el proyecto se logró involucrar a distintos grupos etéreos hacia mayor sensibilización y preparación para la vejez y el envejecimiento. De esta forma se dieron las condiciones para la construcción de una sociedad para todas las edades.

\section{Conclusiones}

Dentro de un marco de creciente envejecimiento es fundamental generar condiciones para el desarrollo de relaciones sociales que contribuyan a una sociedad para todas las edades.

Mediante esta iniciativa de incentivar las relaciones intergeneracionales, se logró visitar alrededor de 15 hogares de ancianos, 10 centros diurnos, 15 centros educativos y dos centros penales; supervisar el servicio de transporte de 
exclusividad para personas adultas mayores y crear una oferta de cursos tanto de sensibilización como de capacitación a personas de todas las edades, lo cual constituyó parte del paquete que contenía esta propuesta intergeneracional.

A través de estas actividades fue posible fomentar las relaciones intergeneracionales y, de esta forma, dar un paso para corregir la brecha entre las generaciones más jóvenes y las mayores.

El proyecto "Generación Dorada", que arropó el plan de establecer relaciones intergeneracionales fue una propuesta gerontológica que se gestó a finales del año 1999, dentro de un marco de derechos humanos, orientado a fomentar una sociedad más inclusiva, libre de mitos y estereotipos, en aras de erradicar las diversas formas de discriminación y maltrato hacia las personas de edad.

Esta experiencia permitió inculcar valores donde todas las personas tuvieran las mismas oportunidades sin importar la edad; disminuir mitos y estereotipos vinculados a la vejez y al envejecimiento; promover una mejor preparación para la etapa de la vejez; garantizar las condiciones para un envejecimiento activo y darle una resignificación a esta etapa de la vida.

Se logró sensibilizar a los grupos de jóvenes estudiantes, quienes fueron impactados por una realidad que prácticamente la mayoría desconocía, como queda evidenciado en sus testimonios. Se contribuía, así, a inculcar valores de respeto por las personas adultas mayores y, de esta forma, contribuir a modificar las conductas de rechazo que imperaban en muchos jóvenes.

Se promovió que este tipo de actividades fueran propiciadas por más docentes del CEG y, en particular, por quienes tenían a cargo los cursos de la persona adulta mayor.

Por su magnitud, desde inicios del proyecto, se instaba a que otros profesores y profesoras participaran de este tipo de experiencias. Inclusive el mismo estudiantado recomendaba: "que se convirtiera en un requisito este tipo de actividades por la gran necesidad afectiva que hay en estas personas y el gran analfabetismo en los jóvenes sobre el asunto" (Alpízar, 2001, párr. 1).

Fue un programa de educación sobre la vejez y el proceso de envejecimiento, por ello se involucró a los diferentes grupos de edad, y se promovió la defensa de los derechos humanos de las personas adultas mayores. 
Constituye un reto que las relaciones intergeneracionales se logren establecer en un eje fundamental de las políticas públicas en materia de vejez y envejecimiento.

El éxito de un proyecto de esta naturaleza radica en el hecho de que cada vez más estudiantes reciban alfabetización e inducción para mejorar su relación con las personas adultas mayores, como lo expresaron algunos de sus testimonios:

Consideramos tan valios este proyecto cuando no son grandes pensantes, libros gruesos, exposiciones aburridas, exámenes tediosos, sino nuestra experiencia la que nos enseña la importancia de ver lo que estamos haciendo con nuestros viejitos... (Alpízar, 2001, párr. 1)

Por otro lado, resultaba una forma de promover una reducción de la sobrepoblación en los hogares de ancianos y de evidenciar el atropello del cual eran objeto. Mediante una mayor capacitación a las personas de la mediana edad sería posible alcanzar una vejez más saludable y, por ende, más activa.

Sin duda alguna, los programas intergeneracionales generan beneficios para sus participantes, personas adultas mayores, niños, niñas y jóvenes, al propiciar mayores vínculos entre sí.

De hecho, se daban los primeros pasos de los programas intergeneracionales en un momento en los cuales se empezaban a hacer evidentes las huellas del envejecimiento demográfico.

\section{Referencias}

Alpízar, I. (2011). Informe final del proyecto: Generación dorada: Por una mejor calidad de vida de la persona adulta mayor. Heredia, Costa Rica: Centro de Estudios Generales, Universidad Nacional.

Asamblea Legislativa de Costa Rica. (1965). Ley Reguladora del Transporte Remunerado de Personas en Vehículos Automotores. No 3503 de 10-5-1965 Recuperado de http://www.dse.go.cr/es/02ServiciosInfo/Legislacion/PDF/ Sector\%20Transporte/Regulacion/Ley3503TransporteRemunerado.pdf

Consejo Nacional de la Persona Adulta Mayor. (2007). Mitos y estereotipos en el envejecimiento y a vejez. San José, Costa Rica: CONAPAM.

CONAPAM. (2013). Envejecimiento poblacional (2da ed.). San José, Costa Rica. 
CONAPAM. (2013). Mitos y estereotipos acerca del envejecimiento y la vejez. (2da ed.). San José, Costa Rica.

CONAPAM. (2013). Política Nacional de Envejecimiento y Vejez 2011-2012. San José, Costa Rica.

Newman, S., Sánchez, M. (2007). Los programas intergeneracionales. Revista Programas intergeneracionales: Hacia una sociedad para todas las edades. Recuperado de http://www.aepumayores.org/sites/default/files/Programas Intergeneracionales_Coleccion_Estudios_Sociales_vol23_es.pdf

Organización de Naciones Unidas. (1982). Primera Asamblea Mundial sobre el Envejecimiento. Viena, Austria: ONU.

Organización de Naciones Unidas. (2002). Informe de la Segunda Asamblea Mundial sobre el Envejecimiento. Madrid, España: ONU.

Organización de Naciones Unidas (2003). Declaración Política y Plan de Acción Internacional de Madrid sobre el Envejecimiento. Segunda Asamblea Mundial sobre envejecimiento 2002. Madrid, España. Recuperado de http://social. un.org/ageing-working-group/documents/mipaa-sp.pdf

Relaciones intergeneracionales (2009). Informe sobre la situación en siete países de Iberoamérica. Revista Enlace en Red. Recuperado de http://www.aepumayores.org/sites/default/files/Envejecimiento_Activo_Relaciones_Intergeneracionales.pdf 